\title{
EDITORIAL
}

\section{Commentaries section enhanced}

Hypertension Research (2013) 36, 477; doi:10.1038/hr.2013.32

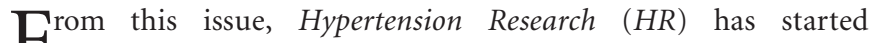
F publishing commentaries on one or more recently published, hypertension-related research articles that are of interest to our readers. This is a new addition to the section of existing commentary articles in $H R$ that are based on the researches published in this journal. We think it is a good complement and will make HR's commentary section more attractive and useful to readers. Commentary articles are usually commissioned from recognized experts, and we ask authors to provide a balanced overview of the field and not to focus on their own work or that of their close colleagues.

We hope you will find HR's newly enhanced commentaries section interesting. Your comments and suggestions are always welcome.

Shokei Kim-Mitsuyama Editor-in-Chief Hypertension Research 